Bangladesh Journal of Neuroscience 2013; Vol. 29 (1) : 24-30

\title{
Carotid atherosclerosis in diabetic patients with Ischemic Stroke: an experience at BIRDEM
}

\author{
NIRMALENDU BIKASH BHOWMIK ${ }^{1}$, MD. AZIZUL HAQUE ${ }^{2}$, MD. SAIFUDDIN ${ }^{3}$ \\ MD. RASHEDUL ISLAM ${ }^{4}$, RUMANA HABIB $^{5}$, AMINUR RAHMAN ${ }^{5}$ \\ ZAHID HASSAN ${ }^{7}$, MD. AMIRUL HAQUE ${ }^{8}$
}

\begin{abstract}
:
Background and objectives: Carotid atherosclerosis constitutes an important cause of ischemic stroke. Type 2 diabetes mellitus is known to be an independent risk factor for stroke and its recurrence. This study was aimed to explore relationship between carotid atherosclerosis and ischaemic stroke in patients with diabetes and its association with other risk factors. Materials and Methods: A total number of 50 ischemic stroke patients, as confirmed by CT/MRI, patients with type 2 diabetes mellitus, of both sexes, age range 40-79 years, were recruited in the study from in-patient Neurology department, BIRDEM. Carotid duplex study was done. Ischemic Stroke patients were sub grouped into normal, mild (<2), moderate (2-4) and severe (>4) on the basis of ICA/CCA flow velocity ratio. Blood glucose and fasting lipid levels and blood pressure were recorded. Lipid abnormality ,LDL 130mg/dl, total cholesterol 200 mg/dl was defined following NCEP and AHA guidelines. Data were collected in a pre-formed printed case record form and analyzed using SPSS software. Results: Of the 50 cases only $9(18 \%)$ had normal ICA/CCA flow velocity ratio. Their mean age was $48.0 \pm 4.4$ years. Three (6\%) cases had severe form of ICA/CCA flow velocity ratio $47.67 \pm 2.08$. Subjects with stroke having mild to moderate compromised flow velocity ratio were significantly older $(p<0.001)$. Mean $( \pm S D)$ total cholesterol and $L D L-$ cholesterol were significantly associated with ICA/CCA flow velocity ratio ( $p<0.001$ for both) in the study subjects. Serum triglyceride did not show significant association with ICA/ CCA flow velocity ratio. Uncontrolled diabetes mellitus $(p=0.02)$ and blood pressure $(p=0.001)$ shown to be significantly associated with atherosclerotic changes in the study subjects. Conclusions: The data conclude that diabetic patients with ischemic stroke have carotid atherosclerosis which is significantly related with lipid abnormality. High total cholesterol and LDL cholesterol are associated with degree of carotid atherosclerosis. Study subjects with severe atherosclerotic changes are relatively younger in age. Carotid duplex study should be planned in ischemic stroke patients with diabetes which will identify the individual at risk and suggest them necessary prevention program.
\end{abstract}

Keywords: Diabetes mellitus, ischemic stroke, carotid atherosclerosis, lipid abnormality.

\section{Introduction:}

Stroke is the third leading cause of death and disability in western countries. The very basic vascular pathology remained to be atherosclerotic changes and resulting stenosis of the internal carotid artery ${ }^{1,2}$. Stenosis is suggested to alter the perfusion to the brain owing to reduction of diameter of the carotid artery or leaving potential site for thrombi originating from plaques at the site of stenosis ${ }^{3}$.

Cardiovascular disease is the leading cause of morbidity and mortality in patients with diabetes,

1. Associate Professor of Neurology, BIRDEM General Hospital \& Ibrahim Medical College, Shahbag, Dhaka

2. Resident (Neurology), BSMMU, Dhaka

3. Assistgant Registrar, Neurology Unit II, BIRDEM General Hospital \& Ibrahim Medical College, Shahbag, Dhaka

4. Registrar, Neurology, BIRDEM General Hospital \& Ibrahim Medical College, Shahbag, Dhaka

5. Registrar, Neurology, BIRDEM General Hospital \& Ibrahim Medical College, Shahbag, Dhaka

6. Associate Professor of Neurology, BIRDEM General Hospital \& Ibrahim Medical College, Shahbag, Dhaka

7. Associate Professor, Dept. of Physiology \& Molecular Biology, BIRDEM, Shahbag, Dhaka

8. Professor of Neurology, BIRDEM General Hospital \& Ibrahim Medical College, Shahbag, Dhaka 
who also have twice the risk of stroke or heart disease and a greater prevalence of atherosclerosis than patients than who are not diabetic ${ }^{4}$. Diabetes mellitus plays a crucial role as an independent risk factor for stroke in $37-42 \%$ of cases, especially among patients younger than $65^{5}$ years. It has also been determined that the increased frequency of dyslipidaemia, hyperglycaemia, obesity, hypertension and associated nephropathy may contribute to accelerated atherogenesis in diabetic subjects ${ }^{6}$. Epidemiological data have demonstrated that the risk of coronary heart disease and other forms of atherosclerotic vascular diseases rise in those with high plasma cholesterol level and in particular the high ratio of total cholesterol to LDLcholesterol. A much weaker correlation also found to exist with plasma triglyceride level. Extensive large-scale randomized trials have shown that LDL and total cholesterol level below the target values reduces the risk of cardiovascular events including death, myocardial infarction and stroke, and also reduces the need for revascularization ${ }^{7}$.

Identification of carotid stenosis and addressing the risk factors with appropriate medical intervention claimed to reduce the risk of stroke in diabetic patients. Duplex ultrasonography of the carotids is a useful diagnostic tool for the detection of carotid stenosis and its diagnostic value increases in individuals with presence of other risk factors. Although diabetes is a recognized risk factor for ischemic stroke, data are lacking about the involvement of carotid artery stenosis in stoke patient with diabetes mellitus. Hence the present study was undertaken to evaluate internal carotid artery and common carotid artery flow velocity ratio and explore its relationship in diabetic patients with ischaemic stroke.

\section{Materials and Methods:}

This cross-sectional observational study was conducted in the Department of Neurology, BIRDEM. Fifty (50) ischemic stroke patients of both sexes, confirmed by imaging (CT/MRI), with type 2 diabetes mellitus, age range 40-79 yrs, not on lipid lowering agents, were consecutively recruited in the study during the period of June 2010 to December 2010. Patients with hemorrhagic stroke, other complications and absence of diabetes were excluded. Written consent was obtained from close relations of the patient(s). Duplex study of neck vessels was performed and subjects were classified as normal (no alteration of flow velocity), mild $(<2)$, moderate (2-4) and severe (>4) carotid atherosclerosis on the basis of ICA/CCA flow velocity ratio ${ }^{8}$. Blood glucose, fasting lipid levels and blood pressure were recorded. Total cholesterol and LDL cut-off values were $200 \mathrm{mg} / \mathrm{dl}$ and 130 $\mathrm{mg} / \mathrm{dl}$ respectively following National Cholesterol Education Program (NCEP) and American Heart Association (AHA) guidelines. Variables of interest for the study were recorded in a predesigned case record form. Statistical analyses were performed using Statistical Package for Social Science (SPSS) Version 15 for Windows. Data were expressed as mean $\pm S D$ and number (percent) as appropriate. One way ANOVA were performed as applicable. $P$ value $<0.05$ was taken as level of significance.

\section{Results:}

Of the 50 study subjects $28(56.0 \%)$ were male and $22(44.0 \%)$ female. Distribution of male female subjects on the basis of ICA/CCA flow velocity was shown in table 1 . The distribution did not show significant association $(p=0.37)$.

Of the 50 subjects $18 \%, 52 \%, 24 \%$ and $6 \%$ had normal, mild, moderate and severe carotid atherosclerotic disease as judged by ICA/CCA flow velocity (Table 2$)$. Mean $( \pm S D)$ age (yrs) of subgroup of patients was $48.00 \pm 4.44,56.31 \pm 4.36$, $58.50 \pm 8.47$ and $47.67 \pm 2.08$ respectively. This mean age distribution showed significant association $(p<0.001)$.

Mean ( $\pm S D$ ) triglyceride levels of the study subjects was $194 \pm 52$. In the four subgroups the values were $163 \pm 10,196 \pm 59,217 \pm 52$ and $175 \pm 13$ which did not show any statistical significant association (Table 3).

Mean $( \pm S D)$ total cholesterol level of the study subjects was $236 \pm 38$. In the different subgroups on the basis of ICA/CCA flow velocity mean total cholesterol level was significantly higher (Table 3). The value was $176 \pm 14,233 \pm 13,275 \pm 15$ and $296 \pm 12$ for normal, mild, moderate and severe groups respectively $(p<0.001)$ (Table 3$)$. 
Mean $( \pm S D)$ LDL cholesterol of the total subjects was $124 \pm 22$. In the different subgroups on the basis of ICA/CCA flow velocity mean total cholesterol level was significantly higher. The value in the four subgroups was $105 \pm 21,109 \pm 14,144 \pm 10$ and $157 \pm 6$ respectively $(p<0.001)$ (Table 3$)$

Of the 50 in 17 subjects blood pressure was controlled and of them ICA/CCA flow velocity was normal in $8(47 \%)$ and $6(35.3 \%)$ and $3(17.6 \%)$ mild and moderate atherosclerosis disease. Among those with uncontrolled blood pressure ICA/CCA flow velocity was normal in 2 (3\%). And 20 (60.6\%), $9(27.3 \%)$ and $3(9.1 \%)$ patients had mild moderate and severe atherosclerosis (Table 4). This distribution showed statistical significant association $(p=0.001)$ (Table4).

Of the 50 subjects diabetes was in control among $18(34.6 \%)$ and uncontrolled among 32 (53.6\%) cases. Of the 18 subjects with controlled diabetes 7 $(38.9 \%)$ had normal ICA/CCA flow velocity and 7 $(38.9 \%)$ mild and $4(22.2 \%)$ moderate atherosclerotic disease. Of the 32 patients with uncontrolled diabetes mellitus $2(6.3 \%)$ had normal ICA/CCA flow velocity and 19 (59.4\%), 8 (25\%) and $3(9.4 \%)$ had mild, moderate and severe atherosclerotic disease respectively $(p=0.02)$ (Table 5$)$.

Table-I

Male female distribution of the study subjects $(n=50)$

\begin{tabular}{lccc}
\hline ICA/CCA flow velocity ratio & \multicolumn{2}{c}{ Gender } & P value \\
\cline { 2 - 3 } & Male & Female & \\
\hline Normal & $3(6.0 \%)$ & $6(12.0 \%)$ & 0.37 \\
Mild $(<2)$ & $17(34.0 \%)$ & $9(18.0 \%)$ & \\
Moderate $(2-4)$ & $6(12.0 \%)$ & $6(12.0 \%)$ & \\
Severe $(>4)$ & $2(4.0 \%)$ & $1(2.0 \%)$ & \\
\hline Total & $28(56.0 \%)$ & $22(44.0 \%)$ & \\
\hline
\end{tabular}

Results were expressed as number (percent).

Chi-square test was performed to calculate statistical association. $P$ value $<0.05$ was taken as level of significance.

Table-II

Distribution of study subjects on the basis of ICA/CCA flow velocity and age of the subgroups ( $n=50)$

\begin{tabular}{lccc}
\hline ICA/CCA flow velocity ratio & No $(\%)$ & Age (yrs) & p value \\
\hline Normal & $9(18 \%)$ & $48.00 \pm 4.44$ & \\
Mild $(<2)$ & $26(52 \%)$ & $56.31 \pm 4.36$ & $<0.001$ \\
Moderate $(2-4)$ & $12(24 \%)$ & $58.50 \pm 8.47$ & \\
Severe $(>4)$ & $3(6 \%)$ & $47.67 \pm 2.08$ & \\
\hline
\end{tabular}

Results were expressed mean $\pm S D$ and number (percent) where appropriate. One way ANOVA test was performed to calculate significant association. $P$ value $<0.05$ was taken as level of significance.

Table-III

Serum triglyceride, total cholesterol and LDL-cholesterol of the study subjects $(n=50)$

\begin{tabular}{lccc}
\hline ICA/CCA flow velocity ratio & Triglyceride $(\mathrm{mg} / \mathrm{dl})$ & Total cholesterol $(\mathrm{mg} / \mathrm{dl})$ & LDL-c $(\mathrm{mg} / \mathrm{dl})$ \\
\hline Normal $(\mathrm{n}=9)$ & $163 \pm 10$ & $176 \pm 14$ & $105 \pm 21$ \\
Mild $(<2)(\mathrm{n}=26)$ & $196 \pm 59$ & $233 \pm 13$ & $109 \pm 14$ \\
Moderate $(2-4)(\mathrm{n}=12)$ & $217 \pm 52$ & $275 \pm 15$ & $144 \pm 10$ \\
Severe $(>4)(\mathrm{n}=3)$ & $175 \pm 13$ & $296 \pm 12$ & $157 \pm 6$ \\
$P$ value & 0.12 & $<0.001$ & $<0.001$ \\
\hline
\end{tabular}

Results were expressed as mean \pm SD. One way ANOVA test was performed to calculate significant association. $P$ value $<0.05$ was taken as level of significance. 
Table-IV

Distribution of the study subjects on the basis of ICA/CCA flow velocity ratio and blood pressure $(n=50)$

\begin{tabular}{lccc}
\hline ICA/CCA flow velocity ratio & \multicolumn{2}{c}{ Blood pressure } & P value \\
\cline { 2 - 3 } & Controlled (17) & Uncontrolled (33) & \\
\hline Normal $(n=9)$ & $8(16.0 \%)$ & $1(2.0 \%)$ & 0.001 \\
Mild $(<2)(n=26)$ & $6(12.0 \%)$ & $20(40.0 \%)$ & \\
Moderate $(2-4)(n=12)$ & $3(6.0 \%)$ & $9(18.0 \%)$ & \\
Severe $(>4)(n=3)$ & 0 & $3(6.0 \%)$ & \\
\hline
\end{tabular}

Data were expressed as number percent). Chi-square test was performed to calculate statistical association. $P$ value $<0.05$ was taken as level of significance.

Table-V

Distribution of the study subjects on the basis of ICA/CCA flow velocity ratio and diabetes control $(n=50)$

\begin{tabular}{|c|c|c|c|}
\hline \multirow[t]{2}{*}{ ICA/CCA flow velocity ratio } & \multicolumn{2}{|c|}{ Diabetes } & \multirow[t]{2}{*}{$P$ value } \\
\hline & Controlled (18) & Uncontrolled (32) & \\
\hline Normal $(n=9)$ & $7(14 \%)$ & $2(4 \%)$ & \\
\hline Mild $(<2)(n=16)$ & $7(14 \%)$ & $19(38 \%)$ & 0.02 \\
\hline Moderate $(2-4)(n=12)$ & $4(8 \%)$ & $8(16 \%)$ & \\
\hline Severe $(>4)(n=3)$ & 0 & $3(6 \%)$ & \\
\hline
\end{tabular}

Data were expressed as number percent). Chi-square test was performed to calculate statistical association. $P$ value $<0.05$ was taken as level of significance.

\section{Discussion:}

Gender bias found to exist in the prevalence of atherosclerotic lesions in the extracranial carotid artery; $4.4 \%$ of all the subjects, $7.9 \%$ of the men, and $1.3 \%$ of the women had atherosclerosis accompanied by stenosis of $>50 \%$ were found. A strong association between these lesions and the results of a $75 \mathrm{~g}$ oral glucose tolerance test was found in both sexes ${ }^{9-12}$. Though in other study male gender was found as an independent predictor of carotid atherosclerosis and female gender was found protective ${ }^{13}$. However, no statistical difference was observed regarding frequency of ischemic stroke in the present study.

Among 50 study subjects $82 \%$ of the subjects with stroke had mild to severe form of atherosclerosis and $18 \%$ had normal flow velocity. The degree of stenosis has been recognized as an important risk factor of stroke ${ }^{14}$.

The study showed that in case of mild atherosclerotic disease $12 \%$ patient had controlled and $40 \%$ uncontrolled HTN In case of moderate atherosclerotic disease $6 \%$ patients show controlled HTN, $18 \%$ uncontrolled HTN. In case of severe atherosclerotic disease none of the patients had controlled blood pressure. In case of subjects with normal flow ratio $16 \%$ had controlled and $2 \%$ uncontrolled blood pressure. In case of mild atherosclerotic disease $14 \%$ had controlled and $38 \%$ uncontrolled DM. In case of moderate atherosclerotic disease $8 \%$ patients show controlled DM, $16 \%$ patients show uncontrolled DM. In case of severe atherosclerotic disease $0 \%$ patient show controlled DM, $6 \%$ patient uncontrolled DM. In case of subjects with normal flow ratio $14 \%$ had controlled DM, $4 \%$ patients show uncontrolled DM.

Total cholesterol and LDL- cholesterol were found to be associated with degree of stenosis. Similar trend was found with uncontrolled blood pressure and diabetes. Our findings are consistent with literature on risk factors associated with carotid stenosis $^{15}$. Higher prevalence of carotid 
atherosclerosis in diabetic subjects compared with non diabetic subjects was shown by other investigators ${ }^{16}$.

Increased carotid intimal-medial thickness (IMT) is associated with cardiovascular risk factors and is predictive of stroke in older adults. In men, LDL cholesterol, HDL-cholesterol and diastolic blood pressure were predictive of carotid IMT in a risk factor load model, whereas in women, LDL cholesterol, body mass index, and triglycerides were predictive ${ }^{17-21}$.

Intervention trials demonstrated that correction of risk factors, like TG, LDL-C and HDL-C, results in a reduced risk of stroke, at least ishaemic stroke in patients with established IHD. Low levels of HDL-C are common among patients after an ischemic stroke, especially in those with a higher degree of carotid stenosis $22-25$. Multiple regression analysis revealed that carotid atherosclerosis had significant relationships with age, systolic blood pressure, fasting blood glucose, pack-years of smoking, total serum cholesterol, and HDL cholesterol in men $(p<0.05)$ and with age, systolic blood pressure, packyears of smoking, and total serum cholesterol in women $(p<0.05)$. Cardiovascular risk factors were strongly related to carotid atherosclerosis and that the proportion of severe carotid atherosclerosis with $>50 \%$ stenosis was not low and was almost equal to that reported in developed western countries ${ }^{9-12}$.

Interesting finding of the present study is that relatively younger subjects suffering from severe carotid atherosclerotic disease as observed by severe restriction of ICA/CCA flow velocity ratio. These subjects are also found to have higher mean total cholesterol and LDL cholesterol level. These later scenario however reconfirm that lipid abnormalities plays an important role in the pathogenesis of atherosclerosis. This study also demonstrated that uncontrolled diabetes mellitus and blood pressure putting additional risks in the vascular pathological process. It is understood that environmental factors play crucial role in the pathogenesis of atherosclerotic disease in the presence of genetic susceptibility. It has also been shown that good metabolic control can prevent or at least delay the atherosclerotic process not only among diabetic but also of the non diabetic subjects.

Routine health check is not practiced in our country. Diabetic patient with ischemic stroke having severely restricted ICA/CCA flow velocity ratio are relatively young. The pathological process, however, suggested to be started much earlier. A regular follow up would have identified the individuals at risk and appropriate measures might have helped in delaying the ongoing process providing them better quality of life. The notable limitation of the present study is the lack of data regarding socioeconomic status which could have assisted in explaining their lifestyle, food habit and above all the socioeconomic transformation.

\section{Conclusion:}

Ischemic stroke patients with diabetes have carotid atherosclerosis and they have significant relationship with lipid abnormality. High total cholesterol and LDL cholesterol are associated with degree of carotid atherosclerosis. Study subjects with severe atherosclerotic changes are relatively younger, awareness about the risk factors may help susceptible individuals to modify lifestyle and take medication to prevent or at least delay the events. In the management and investigation plan carotid duplex study should be planned which will identify the individual at risk and suggest them necessary prevention program.

\section{Acknowledgement:}

The authors of the article express sincere gratitude to relatives of the patients for consenting and allowing us to conduct the study. The authors also thankful to the support staff in the ward to extend their help in conducting the study.

\section{References:}

1. Lacroix $\mathrm{P}$, Aboyans $\mathrm{V}$, Criqui $\mathrm{MH}$, Bertin $\mathrm{F}$, Bouhamed T, Archambeaud F et al. Type -2 diabetes and carotid stenosis: a proposal for a screening strategy in asymptomatic patients. Vascular Medicine. 2006;11:93-99

2. Park JH, Kim WH, Kim Jh, Park TS, Baek HS. Prevalence of and risk factors for extracranial internal carotid artery stenosis in Korean type 2 diabetic patients. Diabetic Medicine. 2006; 23:1377-80 
3. Kisten Y, Govender P, Naidoo NG, Gihwala D, Isaac F. Duplex ultrasound: A diagnostic tool for carotid stenosis management in type 2 diabetes mellitus. Afr j Prm Health Care Fem Med.2013; 5(1): 414-20

4. Buse Jb, Ginsberg HN, Bakris GL, Clark NG, Costa F, Eckel R et al., Primary prevention of cardiovascular diseases in people with diabetes mellitus: a scientific statement from the American Heart Association and the American Diabetes Association. Diabetes Care. 2007; 30:162-72

5. MaitiR, Agarwal NK. Atherosclerosisi in Diabetes Mellitus:Role of Inflammation. Indi. J. Med Sci 2007; 61(8): 292-306.

6. Paulose KP. Carotid Artery Disease in type 2 Diabetes patients. Int J Diab Dev Countries 2002; (22):133-34.

7. Bloomfield P, Bradbury A, Grubb NR, Newby DE. Atherosclerosis. In: Davidson's Principles and Practice of Medicine, $21^{\text {Th }} \mathrm{Ed}$, Nicholas AB, Ncki RC, John AA Hunters (Eds) Churchill Livingstone, 2010; 18: 577-79.

8. Grant EG, Benson CB, Moneta GL, Alexandrov AV, Baker JD, Bluth El et al. Carotid artery stenosis: gray-scale and Doppler US diagnosis-Society of Radiologists in Ultrasound Consensus Conference. Radiology. 2003; 229(2):340-46.

9. Randomised trial of cholesterol lowering in 4444 patients with coronary heart disease: the Scandinavian Simvastatin Survival Study (4S). Lancet 1994; 19; 344(8934): 1383-89.

10. Prevention of cardiovascular events and death with pravastatin in patients with coronary heart disease and a broad range of initial cholesterol levels. The Long-Term Intervention with Pravastatin in Ischaemic Disease (LIPID) Study Group. N Engl J Med 1998; 339 (19):1349-57.

11. Kieffer SA, Takeya Y, Resch JA, Amplatz K. Racial differences in cerebrovascular disease: angiographic evaluation of Japanese and American population. AJR Am J Roentgenol. 1967; 101: 94-99.
12. Tomita $\mathrm{T}$, Mihara $\mathrm{H}$. Cerebral angiographic study on CVD in Japan. Angiology 1972; 23: 228-39.

13. Matheisen EB, Joakimsen O, Bonna K. Prevalence of risk factors associated with carotid artery stenosis: The Troms study. Cerebrovasc Dis. 2001; 12:44-51

14. Matheisen EB, Joakimsen O, Bonna K. Echoluent plaques are associated with high risk of ischaemic cerebrovascular events in carotid stenosis. The Troms study. Circulation 2001; 103: 2171-75

15. North American Symptomatic Carotid Endarterectomy Trial Collaborators. Beneficial effect of carotid endartectomy in symptomatic patients with high grade stenosis. N.Eng $\mathrm{J}$ Med. 1991; 325:445-52

16. De Angelis $M$, Scrucca $L$, Leandri $\mathrm{M}$, Mincigrucci S, Bistoni S, Bovi M, et al. Prevalence of carotid stenosis in type 2 diabetic patients asymptomatic for cerebrovascular disese. Diabetes Nutr Metab 2003; 16:48-55

17. Heiss G, Sharrett AR, Barnes R, Chambless LE, Szklo M, Alzola C et al. Carotid atherosclerosis measured by B-mode ultrasound in populations: associations with cardiovascular risk factors in the ARIC Study. Am J Epidemiol 1991; 134: 250-56.

18. O'Leary DH, Polak JF, Kronmal RA, Manolio TA, Burke GL, Wolfson SK Jr. Carotid-artery intima and media thickness as a risk factor for myocardial infarction and stroke in older adults. N Engl J Med. 1999; 340: 14-22.

19. Chambless LE, Folsom AR, Clegg LX, Sharrett AR, Shahar E, Nieto FJ et al. Carotid wall thickness is predictive of incident clinical stroke: the Atherosclerosis Risk in Communities (ARIC) Study. Am J Epidemiol. 2000; 151: 478-87.

20. Chambless LE, Heiss G, Folsom AR, Rosamond W, Szklo M, Sharrett AR, et al. Association of coronary heart disease incidence with carotid arterial wall thickness and major risk factors: the Atherosclerosis 
Risk in Communities (ARIC) Study, 19871993. Am J Epidemiol. 1997; 146: 483-94.

21. Burke GL, Evans GW, Riley WA, Sharrett AR, Howard G, Barnes RW et al. Arterial wall thickness is associated with prevalent cardiovascular disease in middle-aged adults: the Atherosclerosis Risk in Communities (ARIC) Study Stroke. 1995; 26: 386-91.

22. Papadakis JA, Mikhailidis DP, Winder AF. Lipids and stroke: neglect of a useful preventive measure? Cardiovasc Res. 1998; 40 (2):265-71.
23. Effect of HMGco A reductase inhibitors on stroke. A meta-analysis of randomized, controlled trials. Ann Intern Med. 1998; 128(2): 89-95.

24. Crouse JR, Byington RP, Furberg CD. HMGCoA reductase inhibitor therapy and stroke risk reduction: an analysis of clinical trials data. Atherosclerosis 1998; 138(1): 11-24.

25. Demchuk AM, Hess DC, Brass LM, Yatsu FM. Is Cholesterol a Risk Factor for Stroke? Yes. Arch Neurol. 1999; 56 (12):1518-20. 